\section{Cureus}

Received 05/28/2019

Review began 05/31/2019

Review ended 05/31/2019

Published 06/07/2019

\section{(c) Copyright 2019}

Patel et al. This is an open access article distributed under the terms of the Creative Commons Attribution License CC-BY 3.0., which permits unrestricted use, distribution, and reproduction in any medium, provided the original author and source are credited.

\title{
The Association of Obstructive Sleep Apnea and Hypertension
}

\author{
Avani R. Patel ${ }^{1}$, Amar R. Patel ${ }^{1}$, Shivank Singh ${ }^{2}$, Shantanu Singh ${ }^{3}$, Imran Khawaja ${ }^{3}$ \\ 1. Internal Medicine, Northern California Kaiser Permanente, Fremont, USA 2. Internal Medicine, \\ Southern Medical University, Guangzhou, CHN 3. Pulmonary Medicine, Marshall University School of \\ Medicine, Huntington, USA
}

$\square$ Corresponding author: Avani R. Patel, avani.94539@gmail.com

Disclosures can be found in Additional Information at the end of the article

\section{Abstract}

Obstructive sleep apnea (OSA) is a condition characterized by recurrent episodes of partial or complete upper airway obstruction during sleep. Hypertension (HTN) is defined by the presence of a chronic elevation of systemic arterial pressure above a certain threshold value $(\geqslant 140 \mathrm{~mm}$ $\mathrm{Hg}$ systolic or $\geqslant 90 \mathrm{~mm} \mathrm{Hg}$ diastolic). On the surface, OSA and HTN appear very different from one another. Despite this, they share several common risk factors including obesity, male gender, and advancing age. In 2003, the Seventh Joint National Committee (JNC VII) recognized OSA as a secondary cause of HTN. As physicians, our goal is to understand the OSAHTN association better through academic study regarding its epidemiology, its pathophysiology, and its treatment.

Categories: Cardiology, Internal Medicine, Pulmonology

Keywords: obstructive sleep apnea, hypertension

\section{Introduction And Background}

Over the years, there has been some overlap between patients of obstructive sleep apnea (OSA) and hypertension (HTN) with about $50 \%$ of HTN patients also having concomitant OSA [1]. As physicians, we see more and more patients who have OSA and either develop HTN or have underlying HTN. Because of this, there is a theory that the two conditions may have a causal, bidirectional relationship [1]. This was proven when OSA was named a secondary cause of HTN by the 2003 Joint National Committee (JNC VII) on prevention, detection, evaluation, and treatment of high blood pressure (BP) [2]. A few years later, the 2019 American Heart Association (AHA) reported the conclusion of a meta-analysis of 27 cohort studies [3]. The meta-analysis determined that severe OSA (apnea/hypopnea index $\geqslant 30$ ) was associated with increased cardiovascular mortality with a hazard ratio of 2.73 (95\% confidence interval [CI], 1.94-3.85) [3]. For this article, the objective is to address the association between OSA and HTN, the epidemiological evidence that supports their causal relationship, the system-specific pathophysiology of OSA that can lead to HTN development, and the treatment of OSA and how it impacts HTN treatment.

OSA is a condition described as recurrent episodes of upper airway inspiratory collapse during sleep, leading to hypopnea (breathing reduction) or apnea (breathing cessation) episodes that cause transient hypoxemia (low oxygen levels in the blood) and hypercapnia (elevated carbon dioxide levels in the blood) [1]. The patient waking from sleep terminates the apneic and hypopneic episodes [1]. The patient then hyperventilates because of the hypoxemia for a brief period of time [1]. These episodes are key to diagnosing the severity of a patients OSA [1]. The 


\title{
Cureus
}

severity is measured by an apnea/hypopnea index (AHI), which measures the number of apnea/hypopnea episodes per hour [1]. The OSA severity is then classified as mild (5-15), moderate (15-30), and severe (30 or more; Table 1) [1]. A patient is diagnosed with OSA when they have an $\mathrm{AHI} \geqslant 5$ events per hour.

\author{
Classification of Obstructive Sleep Apnea \\ Mild \\ $\mathrm{AHI} \geq 5-15$ events per hour \\ Moderate \\ $\mathrm{AHI} \geq 15-30$ events per hour \\ Severe \\ $\mathrm{AHI} \geq 30$ or more events per hour
}

\section{TABLE 1: Classification of obstructive sleep apnea}

Classification of obstructive sleep apnea according to the AHI [1]

AHI, apnea/hypopnea index

HTN is a condition that refers to a sustained increase in BP beyond certain systolic (SBP) and diastolic blood pressure (DBP) levels. The majority of current definitions define HTN as $\mathrm{SBP} \geqslant 140 \mathrm{~mm} \mathrm{Hg}$ and/or DBP $\geqslant 90 \mathrm{~mm} \mathrm{Hg}[4]$. HTN is categorized into essential and secondary. According to an earlier research study by Carretero and Oparil, essential HTN has been defined as high BP in which secondary causes such as renal disease, pheochromocytoma, aldosteronism, or other causes of secondary HTN are not present [5]. The current guidelines for HTN diagnosis are listed in Table 2. The divisions are normal, prehypertension, stage one HTN, and stage two HTN [2].

2003 Joint National Committee Seventh Report on Classification of Blood Pressure in Adults

Classification

Normal

Prehypertension

Stage 1 Hypertension

Stage 2 Hypertension
Systolic Blood Pressure

$<120 \mathrm{~mm} \mathrm{Hg}$

120-139 mm Hg

140-159 mm Hg

$\geq 60 \mathrm{~mm} \mathrm{Hg}$
Diastolic Blood Pressure

and $\quad<80 \mathrm{~mm} \mathrm{Hg}$

or $\quad 80-89 \mathrm{~mm} \mathrm{Hg}$

or $\quad 90-99 \mathrm{~mm} \mathrm{Hg}$

or $\quad \geq 100 \mathrm{~mm} \mathrm{Hg}$

\section{TABLE 2: The 2003 Joint National Committee Seventh Report on Blood Pressure}

\section{Classification}

Current blood pressure classification according to the 2003 Joint National Committee (JNC VII) on prevention, detection, evaluation, and treatment of high blood pressure [2]

\section{Review}




\section{Cureus}

\section{Epidemiology of OSA and HTN}

OSA is a highly prevalent sleep disorder that is estimated to affect $15 \%$ to $24 \%$ of all adults, but that the number is believed to be incorrect because OSA is still greatly underdiagnosed [6]. In 2017, a research study tried to determine the prevalence of OSA in the general adult population and how it varied between different sub-groups [7]. They examined 24 studies and divided the results according to age and AHI. With an $\mathrm{AHI} \geqslant 5$ events per hour, the overall prevalence was between $9 \%$ and $38 \%$ in the general adult population [7-9]. For men having OSA with an AHI $\geqslant 5$, the prevalence ranged from $13 \%$ to $33 \%$ [9-10]. For women having OSA with an AHI $\geqslant 5$, the prevalence ranged from $6 \%$ to $19 \%$ [9-10]. It was also determined that in some advanced age groups, the OSA prevalence was about $84 \%$ [11]. It was even higher in the men, with a $90 \%$ prevalence $[11]$. Moderate OSA (AHI $\geqslant 15$ ) had an adult population prevalence that ranged from $6 \%$ to $17 \%$ but was $36 \%$ in the above 60 years age group [3,8-9,11-12]. Another report stated that a meta-analysis of 27 cohort studies determined that the mild OSA hazard ratio was 1.19 (95\% CI, 0.86-1.65), the moderate OSA hazard ratio was 1.28 (95\% CI, 0.96-1.69), and the severe OSA hazard ratio was 2.13 (95\% CI, 1.68-2.68) and was associated with an all-cause mortality in a dose-response fashion [3].

According to a report from AHA, between 2011 and 2014, the prevalence of HTN in the United States adults was $45.6 \%$ (95\% CI, $43.6 \%$ to $47.6 \%$ ) [3]. This was calculated using the new BP classification from the 2017 American College of Cardiology/AHA guidelines (Table 3) [3,13]. This was in comparison to JNC VII [3]. Based on the 2003 JNC VII classification, the prevalence of HTN was $31.9 \%$ (95\% CI, 30.1\% to $33.7 \%$ ) in the United States adults [2].

\section{American College of Cardiology/AHA Classification of High Blood Pressure}

$\begin{array}{llc}\text { Classification } & \text { Systolic Blood Pressure } & \text { Diastolic Blood Pressure } \\ \text { Normal } & <120 \mathrm{~mm} \mathrm{Hg} & \text { and }<80 \mathrm{~mm} \mathrm{Hg} \\ \text { Elevated Blood Pressure } & 120-129 \mathrm{~mm} \mathrm{Hg} & \text { and }<80 \mathrm{~mm} \mathrm{Hg} \\ \text { Hypertension Stage 1 } & 130-139 \mathrm{~mm} \mathrm{Hg} & \text { or } 80-89 \mathrm{~mm} \mathrm{Hg} \\ \text { Hypertension Stage 2 } & \geq 140 \mathrm{~mm} \mathrm{Hg} & \text { or } \geq 90 \mathrm{~mm} \mathrm{Hg}\end{array}$

\section{TABLE 3: The 2017 American College of Cardiology Classification of High Blood Pressure}

Before diagnosing a patient with hypertension, physicians must base the diagnosis on the average value of more than two BP readings obtained on more than two different occasions [13].

$\mathrm{BP}$, blood pressure

Past research studies have been successful in demonstrating epidemiological evidence of the OSA-HTN relationship. They have determined that OSA is not only a predisposing factor for HTN but there is also a greater incidence of OSA in hypertensive patients [14-15]. OSA is estimated to have a prevalence of $30 \%$ to $50 \%$ in HTN patients [16-17]. In comparison, the prevalence of HTN in OSA patients is between 30\% and 70\% [17]. This is because OSA is underdiagnosed [16-17]. In a previous paragraph, it was mentioned that the JNC VII stated that OSA was a secondary cause of HTN. This was further proven by a 2011 cohort study done in Brazil 


\section{Cureus}

with 125 patients that determined that OSA was the most prevalent secondary cause of elevated $\mathrm{BP}$ in patients [18]. The figure below represents the most prevalent causes of secondary HTN (Figure 1).

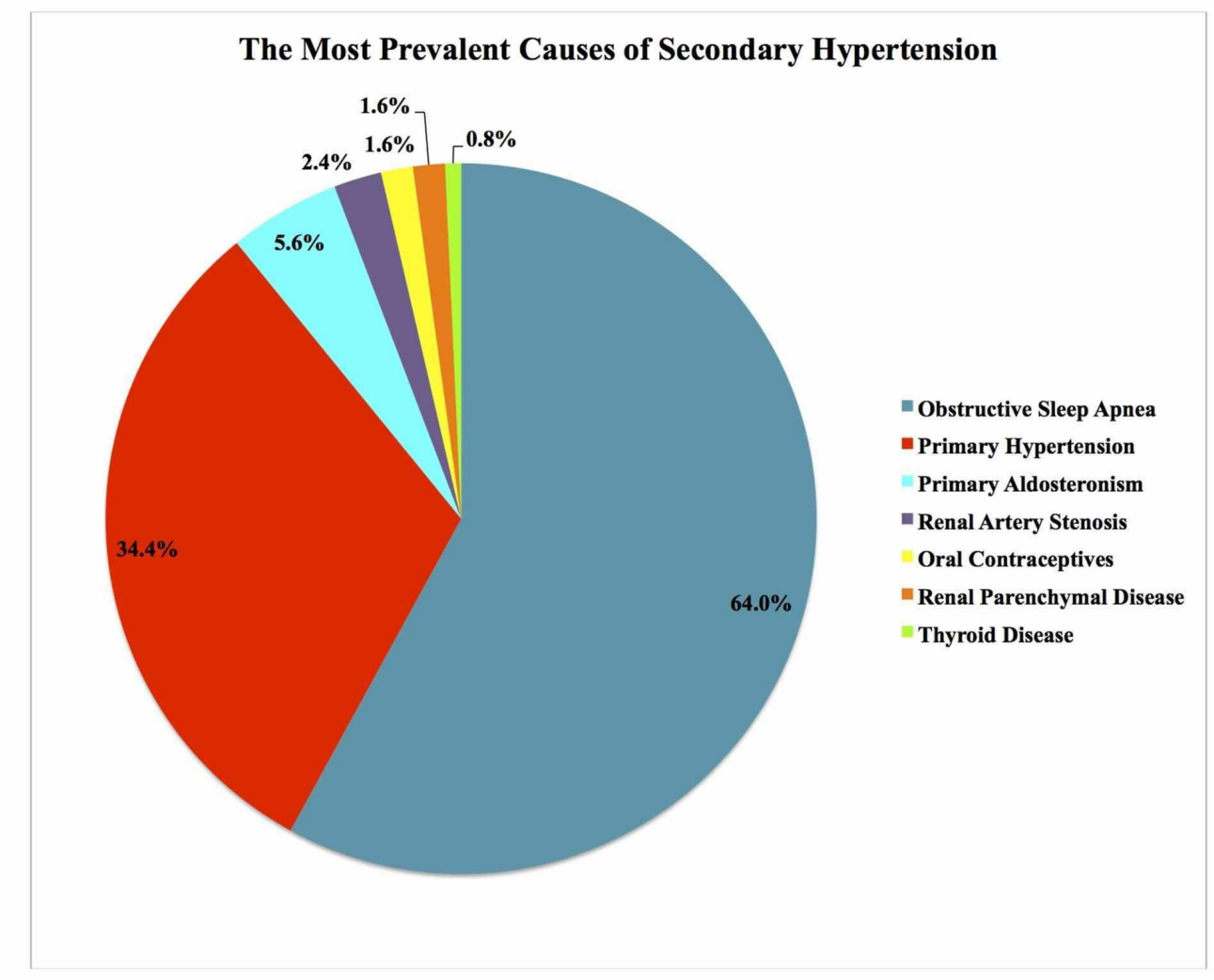

\section{FIGURE 1: The prevalence of secondary causes of hypertension}

A created pie chart representing a 1985 study examining the prevalence of secondary causes of hypertension associated with resistant hypertension in a cohort of 125 patients from Brazil. From this study, it was determined that OSA was the most prevalent secondary cause of resistant hypertension [18].

\section{Pathophysiology}

OSA and HTN are both multifactorial diseases [19]. They share many of the same risk factors (obesity, male gender, and advancing age) [6]. Because of this, and the fact that OSA is the most prevalent secondary cause of HTN, both also share many pathophysiological mechanisms that link them together [18-19]. By understanding these mechanisms as determined by previous research, the development of HTN in OSA cases, and the overall increased risk of cardiovascular disease can be better understood. The figure given below has been created representing different pathophysiological mechanisms linking the two conditions (Figure 2). 


\section{Cureus}

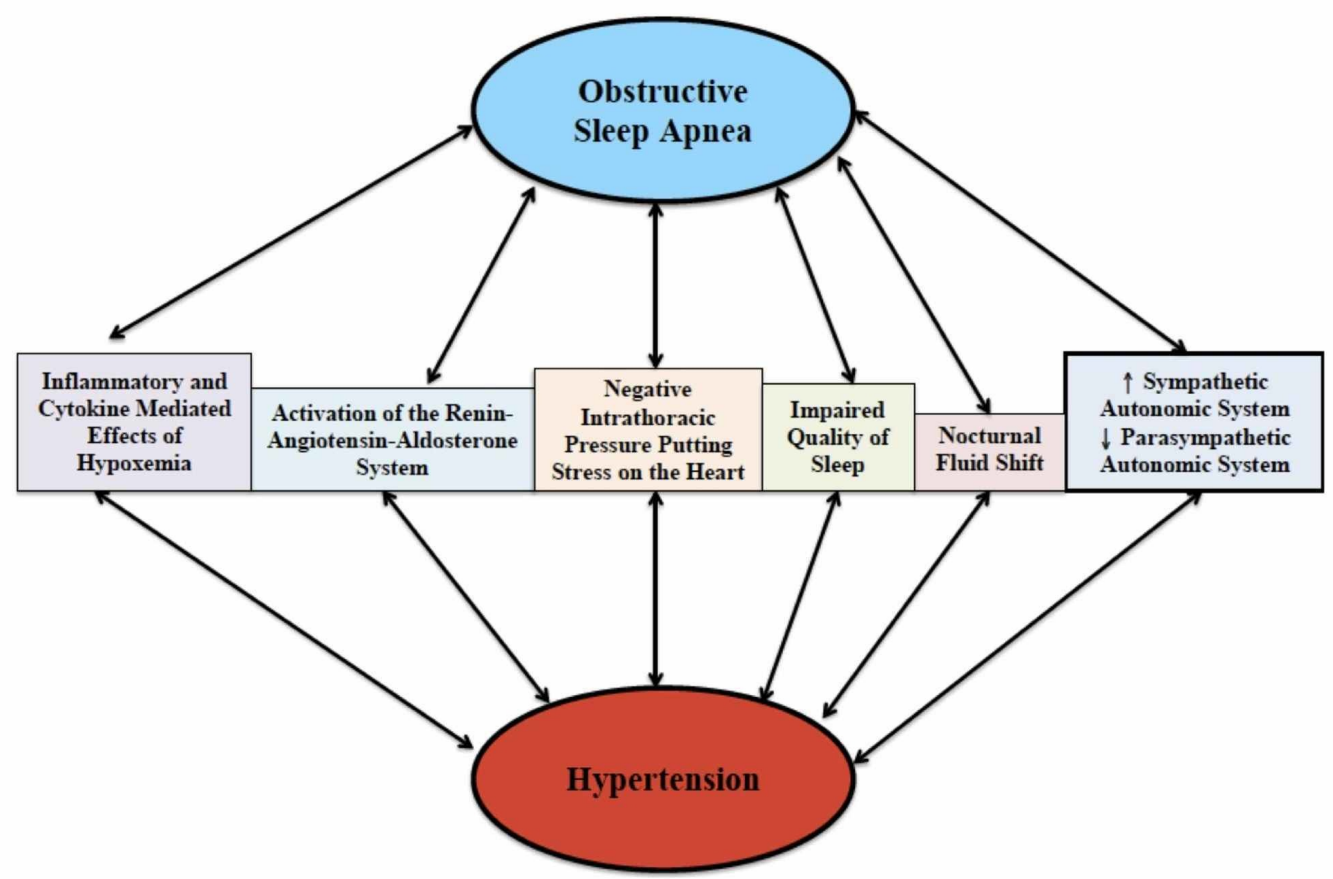

FIGURE 2: The pathophysiological mechanisms shared between obstructive sleep apnea and hypertension

Flow chart representing the shared pathophysiological mechanisms between OSA and HTN

OSA, obstructive sleep apnea; HTN, hypertension

Sleep Inefficiency Due to OSA

Impaired sleep quality (sleep inefficiency and shorter sleep duration) can lead to OSA development [19]. There is decreased sympathetic and increased parasympathetic activity during non-rapid eye movement (NREM) sleep [1]. NREM sleep consists of the majority of sleep time and contributes to the normal circadian variation of BP that leads to a "dipping" of both systolic and diastolic BP at night (decreases by $10 \%$ to 15\%) [20-21]. NREM sleep is followed by rapid eye movement (REM) sleep, which has increased sympathetic activity that leads to transient BP surges [1]. REM sleep is also characterized by generalized skeletal muscle atonia causing an airway collapse in OSA patients especially [1]. This leads to the exacerbation of potential apneic episodes and intensifies the patient's nocturnal sympathetic hyperactivity [1]. A 2012 longitudinal study found that chronic insomnia $(p=0.004)$ and short sleep duration $(p=$ 0.003 ) were both significantly associated with HTN, whereas poor sleep was not $(p=0.756)$ [22].

Nocturnal Fluid Shift in OSA

Another significant pathophysiological mechanism is nocturnal fluid redistribution [19]. During the night, the fluid accumulated in the legs will redistribute to the neck. This is especially significant for OSA and HTN patients in that the reduction of the mean upper airway cross-sectional area can intensify hypopnea/apnea episodes and resultant hypoxia, which will 
ultimately lead to transient BP surges [19]. Friedman et al. (2013) set forth to examine this theory [23]. The theory was proven significant and it was also determined that the upper airway reduction was seen more in resistant HTN patients as compared to controlled HTN patients [23]. OSA also has a prevalence of $83 \%$ in resistant HTN patients [24]. Resistant HTN is defined as a $\mathrm{BP} \geqslant 140 / 90 \mathrm{mmHg}$, while the patient is taking three or more antihypertensive drugs, all of them titrated to the maximum recommended dose [24]. HTN patients would be prone to an increased nocturnal fluid redistribution which worsens their OSA by leading to transient BP surges, which later causes resistant HTN [23].

\section{The Autonomic System Counterregulatory Mechanisms Against Apneic Episodes}

OSA patients have apneic episodes caused by airway collapse during sleep [1]. These episodes lead to transient hypoxemia and hypercapnia that activate the sympathetic autonomic system and down-regulate the parasympathetic autonomic system $[1,25]$. The increased activation of the sympathetic system leads to an increase in catecholamine levels, causing a rise in heart rate and BP that persists into the next day [26]. The rise is most prominent during post-apneic hyperventilation going as high as $240 / 130 \mathrm{~mm} \mathrm{Hg}$ [27-28]. Over time, this sympathetic stimulation can lead to the development of HTN in an OSA patient.

\section{The Inflammatory and Cytokine-mediated Effects of Hypoxemia}

OSA causes intermittent nocturnal hypoxemia and hypercapnia that causes oxidative stress and inflammation [19]. The oxidative stress acts like an ischemic reperfusion injury, leading to the release of reactive oxygen species, inflammatory cytokines (hs-CRP, IL-1, IL-8, IL-6, TNF- $\alpha$, Rantes, and sICAM), and vasoactive substances [29-30]. This leads to an increase in endothelin, a decrease in nitric oxide, vasoconstriction, and endothelial dysfunction [1, 29-30]. Overall, oxidative stress can lead to increased cardiovascular risk.

\section{Negative Intrathoracic Pressure Putting Stress on the Heart}

OSA causes intermittent negative intrathoracic pressure in patients [1]. This pathophysiology combined with OSA nocturnal catecholamine surges can put profound mechanical stress on the heart which can lead to left ventricular hypertrophy and atrial remodeling, thus increasing the risk of heart failure and arrhythmia formation [31].

\section{The Renin-Angiotensin-Aldosterone System}

OSA causes hypoxemia leading to an activation of the renin-angiotensin-aldosterone system (RAAS) [1]. RAAS stimulation increases renin and aldosterone levels (Figure 3) [32]. A 2016 meta-analysis of 13 studies determined that OSA patients had elevated angiotensin II levels compared to control subjects and OSA patients with HTN had higher aldosterone levels compared to normotensive OSA patients [33]. Continuous positive airway pressure (CPAP) therapy is the gold standard of treatment for OSA [1]. CPAP therapy is associated with a downregulation of RAAS activity, leading to consequent BP reduction [34]. From the reduction of BP and the markers, it was concluded that RAAS had a causal role in OSA-mediated HTN [33]. Increased aldosterone caused by RAAS activation can also contribute to fluid retention seen in HTN, which leads to more rostral fluid displacement and an increase in upper airway obstruction $[1,35]$. This will, in turn, worsen the patient's hypoxemia and the pathophysiological cycle will continue. 


\section{Cureus}

The Renin-Angiotensin-Aldosterone System

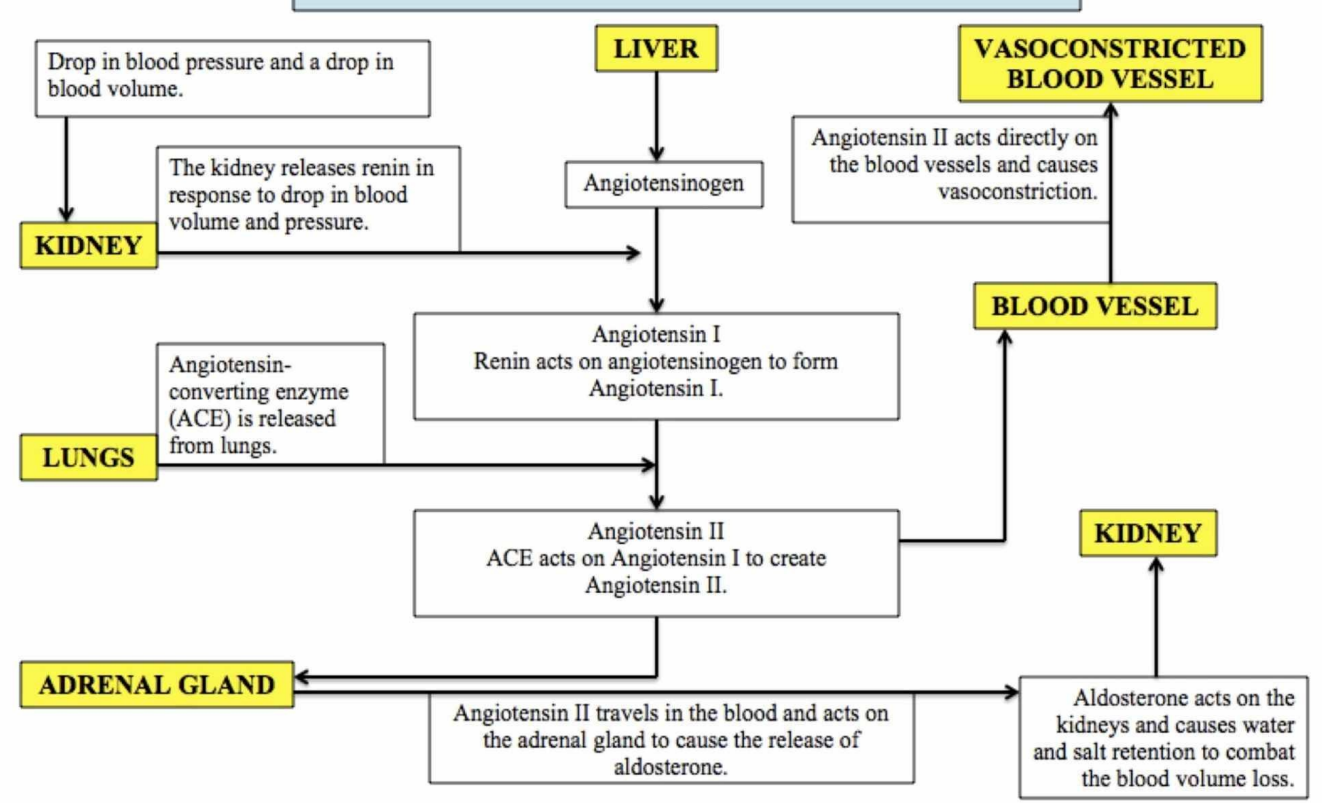

\section{FIGURE 3: The renin-angiotensin-aldosterone system}

Flow chart representing the renin-angiotensin-aldosterone system and the organs and hormones involved in its regulation

\section{Management}

With OSA and HTN, the goals of initial evaluation are to determine the patient's baseline, evaluate for target organ damage, screen for potentially curable causes, identify risk factors that are present, determine the prognosis, and choose a therapy that is specific to the patient's needs [5]. A complete history and physical examination should be done [5]. The patient should also undergo extensive laboratory investigations such as a urine analysis, complete blood count, blood chemistry (potassium, sodium, creatinine, fasting glucose, total and high-density lipoprotein or HDL cholesterol), a 12-lead ECG, creatinine clearance, 24-hour urinary protein, serum uric acid levels, serum calcium, glycosylated hemoglobin, fasting lipid panel, an echocardiography, and plasma renin activity/aldosterone measurements [5].

\section{CPAP Therapy}

For all cases of OSA, CPAP remains the main therapy utilized but its effect on BP reduction has not been proven to be completely effective [1]. In previous clinical trials, CPAP therapy helped reduce the nocturnal sympathetic catecholamine release and their resulting BP surge, but overall BP reduction was not very significant (1.3-3 mm Hg) [36-38]. Despite the modest BP reduction seen in clinical studies, CPAP therapy improves cardiovascular and cerebrovascular health in patients by reducing stroke mortality by $6 \%$ to $8 \%$ and ischemic heart disease mortality by $4 \%$ to $5 \%[2,39]$. 
Obesity is one of the few risk factors commonly seen in both OSA and HTN. Because of this, a reduction in weight can help reduce the OSA severity and BP in an HTN patient. A 2000 cohort study found that a weight gain of $10 \%$ led to a $32 \%$ increase in AHI and a six-fold increase in the odds of developing moderate to severe OSA [40]. In addition, the Wisconsin study involved was also able to determine that a $10 \%$ weight loss would lead to a $26 \%$ AHI decrease [40]. Because OSA is the most prevalent secondary cause of HTN, any decrease in its severity will directly affect HTN severity or development [18].

Antihypertensive Drugs

Antihypertensive medications can be prescribed to all patients with HTN who have mild to moderate OSA (who do not require CPAP therapy) [1]. They can also be given to patients of severe OSA who are either non-tolerant or non-compliant with CPAP therapy [1]. HTN in OSA can occur from catecholamine release from the activated sympathetic system or from the RAAS system activation [1]. Because of this, beta-blockers and aldosterone antagonists may be the best treatment options as they act on these mechanisms [1]. The aldosterone antagonist spironolactone is considered very effective for decreasing the severity of OSA [41]. Another antihypertensive medication called atorvastatin is known to reduce inflammation, which helps reduce the patient's cardiovascular risk [42].

\section{Oral Appliances}

In mild to moderate OSA, oral appliances can be recommended as an alternative treatment to CPAP [1]. A meta-analysis of seven studies (399 OSA patients involved) found that treatment with oral appliances was more beneficial for BP reduction than CPAP therapy [43]. The average drop in the systolic BP and diastolic BP was $2.7 \mathrm{~mm} \mathrm{Hg}(95 \% \mathrm{CI}, 0.8-4.6 ; P=0.04)$ and $2.7 \mathrm{~mm}$ $\mathrm{Hg}$ (95\% CI, 0.9-4.6; $P=0.004$ ), respectively [43].

\section{Upper Airway Surgery}

Upper airway surgery is also a treatment option for OSA patients who require BP reduction [1]. These options include tonsillectomy and uvulopalatopharyngoplasty (UPPP) [1]. A 2010 randomized controlled trial determined that modified UPPP significantly improved sleepiness, nocturnal respirations, and quality of life [44]. The trial also determined that the BP was reduced significantly after surgery in a select group of patients with moderate to severe OSA [44].

\section{Conclusions}

More and more OSA patients present or need treatment for co-existing HTN. The OSA airway collapse leads to the BP being driven up, and without treatment, the patient continues in a selfperpetuating pathophysiological cycle that leads to an increase in their cardiovascular and cerebrovascular risk. Although enormous collected data and treatments are available for OSA requiring BP reduction, more strategies are critically needed. The only way to make this happen is to create a path for more research and larger clinical trials. As physicians, we must spearhead this cause because more and more of our patients will come in with this condition.

\section{Additional Information}

\section{Disclosures}

Conflicts of interest: In compliance with the ICMJE uniform disclosure form, all authors declare the following: Payment/services info: All authors have declared that no financial support was received from any organization for the submitted work. Financial relationships: 
All authors have declared that they have no financial relationships at present or within the previous three years with any organizations that might have an interest in the submitted work. Other relationships: All authors have declared that there are no other relationships or activities that could appear to have influenced the submitted work.

\section{References}

1. Ahmad M, Makati D, Akbar S: Review of and updates on hypertension in obstructive sleep apnea. Int J Hypertens. 2017, 2017:1-13. 10.1155/2017/1848375

2. Lenfant C, Chobanian AV, Jones DW, Roccella EJ: Seventh report of the Joint National Committee on the Prevention, Detection, Evaluation, and Treatment of High Blood Pressure (JNC 7): resetting the hypertension sails. Hypertension. 2003, 41:1178-9. 10.1161/01.HYP.0000075790.33892.AE

3. Benjamin EJ, Muntner P, Alonso A, et al.: Heart Disease and Stroke Statistics-2019 Update: a report from the American Heart Association. Circulation. 2019, 139:e56-e528. 10.1161/CIR.0000000000000659

4. Chobanian AV: Guidelines for the management of hypertension. Med Clin North Am. 2017, 101:219-227. 10.1016/j.mcna.2016.08.016

5. Carretero OA, Oparil S: Essential hypertension. Part I: definition and etiology. Circulation. 2000, 101:329-35. 10.1161/01.CIR.101.3.329

6. Young T, Peppard PE, Gottlieb DJ: Epidemiology of obstructive sleep apnea: a population health perspective. Am J Respir Crit Care Med. 2002, 165:1217-39. 10.1164/rccm.2109080

7. Senaratna CV, Perret JL, Lodge CJ, et al.: Prevalence of obstructive sleep apnea in the general population: a systematic review. Sleep Med Rev. 2017, 34:70-81. 10.1016/j.smrv.2016.07.002

8. Tufik S, Santos-Silva R, Taddei JA, Bittencourt LR: Obstructive sleep apnea syndrome in the Sao Paulo epidemiologic sleep study. Sleep Med. 2010, 11:441-6. 10.1016/j.sleep.2009.10.005

9. Reddy EV, Kadhiravan T, Mishra HK, Sreenivas V, Handa KK, Sinha S, Sharma SK: Prevalence and risk factors of obstructive sleep apnea among middle-aged urban Indians: a communitybased study. Sleep Med. 2009, 10:913-8. 10.1016/j.sleep.2008.08.011

10. Redline S, Sotres-Alvarez D, Loredo J, et al.: Sleep-disordered breathing in Hispanic/Latino individuals of diverse backgrounds. The Hispanic Community Health Study/Study of Latinos. Am J Respir Crit Care Med. 2014, 189:335-44. 10.1164/rccm.201309-17350C

11. Heinzer R, Vat S, Marques-Vidal P, et al.: Prevalence of sleep-disordered breathing in the general population: the HypnoLaus study. Lancet Respir Med. 2015, 3:310-8. 10.1016/S22132600(15)00043-0

12. Lee SD, Kang SH, Ju G, et al.: The prevalence of and risk factors for sleep-disordered breathing in an elderly Korean population. Respiration. 2014, 87:372-8. 10.1159/000358442

13. Martínez-Rueda AJ, Olivas-Martínez A, Vega-Vega O, Fonseca-Correa JI, Correa-Rotter R: New 2017 American College of Cardiology/American Heart Association High Blood Pressure Guideline. Hypertension. 2019, 73:142-147. 10.1161/HYPERTENSIONAHA.118.11827

14. Young T, Peppard PE, Gottlieb DJ: Epidemiology of obstructive sleep apnea: a population health perspective. Am J Respir Crit Care Med. 2002, 165:1217-39. 10.1164/rccm.2109080

15. Lavie P, Herer P, Hoffstein V: Obstructive sleep apnoea syndrome as a risk factor for hypertension: population study. BMJ. 2000, 320:479-82. 10.1136/bmj.320.7233.479

16. Fletcher EC, DeBehnke RD, Lovoi MS, Gorin AB: Undiagnosed sleep apnea in patients with essential hypertension. Ann Intern Med. 1985, 103:190-5. 10.7326/0003-4819-103-2-190

17. Silverberg DS, Oksenberg A: Are sleep-related breathing disorders important contributing factors to the production of essential hypertension?. Curr Hypertens Rep. 2001, 3:209-215. 10.1007/s11906-001-0040-8

18. Pedrosa RP, Drager LF, Gonzaga CC, et al.: Obstructive sleep apnea: the most common secondary cause of hypertension associated with resistant hypertension. Hypertension. 2011, 58:811-7. 10.1161/HYPERTENSIONAHA.111.179788

19. Konecny T, Kara T, Somers VK: Obstructive sleep apnea and hypertension: an update. Hypertension. 2014, 63:203-9.

20. Staessen J, Bulpitt CJ, O'Brien E, et al.: The diurnal blood pressure profile. A population study . Am J Hypertens. 1992, 5:386-92. 10.1093/ajh/5.6.386

21. Staessen JA, Fagard RH, Lijnen PJ, Thijs L, Van Hoof R, Amery AK: Mean and range of the 
ambulatory pressure in normotensive subjects from a meta-analysis of 23 studies. Am J Cardiol. 1991, 67:723-7. 10.1016/0002-9149(91)90529-T

22. Fernandez-Mendoza J, Vgontzas AN, Liao D, Shaffer ML, Vela-Bueno A, Basta M, Bixler EO.: Insomnia with objective short sleep duration and incident hypertension: the Penn State Cohort. Hypertension. 2012, 60:929-35. 10.1161/HYPERTENSIONAHA.112.193268

23. Friedman O, Bradley TD, Logan AG: Influence of lower body positive pressure on upper airway cross-sectional area in drug-resistant hypertension. Hypertension. 2013, 61:240-5.

24. Logan AG, Perlikowski SM, Mente A, et al.: High prevalence of unrecognized sleep apnoea in drug-resistant hypertension. J Hypertens. 2001, 19:2271-7.

25. Robertson D, Biaggioni I, Burnstock G, Low P, Paton J: Primer on the Autonomic Nervous System (3rd Edition). Robertson D, Low P, Polinsky R (ed): Elsevier Academic Press, London, UK; 2012.

26. Freet CS, Stoner JF, Tang X: Baroreflex and chemoreflex controls of sympathetic activity following intermittent hypoxia. Auton Neurosci. 2013, 174:8-14. 10.1016/j.autneu.2012.12.005

27. Hedner J, Ejnell H, Sellgren J, Hedner T, Wallin G: Is high and fluctuating muscle nerve sympathetic activity in the sleep apnoea syndrome of pathogenetic importance for the development of hypertension?. J Hypertens Suppl. 1988, 6:529-31.

28. Somers VK, Dyken ME, Skinner JL: Autonomic and hemodynamic responses and interactions during the Mueller maneuver in humans. J Auton Nerv Syst. 1993, 44:253-9. 10.1016/01651838(93)90038-V

29. McNicholas WT: Obstructive sleep apnea and inflammation. Prog Cardiovasc Dis. 2009, 51:392-9. 10.1016/j.pcad.2008.10.005

30. Atkeson A, Yeh SY, Malhotra A, Jelic S: Endothelial function in obstructive sleep apnea . Prog Cardiovasc Dis. 2009, 51:351-62. 10.1016/j.pcad.2008.08.002

31. Bradley TD, Floras JS: Obstructive sleep apnoea and its cardiovascular consequences. Lancet. 2009, 373:82-93. 10.1016/S0140-6736(08)61622-0

32. Patel S, Rauf A, Khan H, Abu-Izneid T: Renin-angiotensin-aldosterone (RAAS): The ubiquitous system for homeostasis and pathologies. Biomed Pharmacother. 2017, 94:317-325. 10.1016/j.biopha.2017.07.091

33. Jin ZN, Wei YX: Meta-analysis of effects of obstructive sleep apnea on the renin-angiotensinaldosterone system. J Geriatr Cardiol. 2016, 13:333-43. 10.11909/j.issn.1671-5411.2016.03.020

34. Nicholl DD, Hanly PJ, Poulin MJ, Handley GB, Hemmelgarn BR, Sola DY, Ahmed SB: Evaluation of continuous positive airway pressure therapy on renin-angiotensin system activity in obstructive sleep apnea. Am J Respir Crit Care Med. 2014, 190:572-80. 10.1164/rccm.201403-05260C

35. Sim JJ, Yan EH, Liu IL, Rasgon SA, Kalantar-Zadeh K, Calhoun DA, Derose SF: Positive relationship of sleep apnea to hyperaldosteronism in an ethnically diverse population. J Hypertens. 2011, 29:1553-9. 10.1097/HJH.0b013e3283492219

36. Ali NJ, Davies RJ, Fleetham JA, Stradling JR: The acute effects of continuous positive airway pressure and oxygen administration on blood pressure during obstructive sleep apnea. Chest. 1992, 101:1526-32. 10.1378/chest.101.6.1526

37. Kario K: Obstructive sleep apnea syndrome and hypertension: ambulatory blood pressure. Hypertens Res. 2009, 32:428-32. 10.1038/hr.2009.56

38. Haentjens P, Van Meerhaeghe A, Moscariello A, De Weerdt S, Poppe K, Dupont A, Velkeniers B: The impact of continuous positive airway pressure on blood pressure in patients with obstructive sleep apnea syndrome: evidence from a meta-analysis of placebo-controlled randomized trials. Arch Intern Med. 2007, 167:757-64. 10.1001/archinte.167.8.757

39. Turnbull F: Effects of different blood-pressure-lowering regimens on major cardiovascular events: results of prospectively-designed overviews of randomised trials. Lancet. 2003, 362:1527-35. 10.1016/S0140-6736(03)14739-3

40. Peppard PE, Young T, Palta M, Dempsey J, Skatrud J: Longitudinal study of moderate weight change and sleep-disordered breathing. JAMA. 2000, 284:3015-21. 10.1001/jama.284.23.3015

41. Gaddam K, Pimenta E, Thomas SJ, Cofield SS, Oparil S, Harding SM, Calhoun DA: Spironolactone reduces severity of obstructive sleep apnoea in patients with resistant hypertension: a preliminary report. J Hum Hypertens. 2010, 24:532-7. 10.1038/jhh.2009.96

42. Totoson P, Fhayli W, Faury G, et al.: Atorvastatin protects against deleterious cardiovascular consequences induced by chronic intermittent hypoxia. Exp Biol Med (Maywood). 2013, 238:223-32. 10.1177/1535370212473696 
Cureus

43. Iftikhar IH, Hays ER, Iverson MA, Magalang UJ, Maas AK: Effect of oral appliances on blood pressure in obstructive sleep apnea: a systematic review and meta-analysis. J Clin Sleep Med. 2013, 9:165-74. 10.5664/jcsm.2420

44. Di Murro A, Petramala L, Cotesta D, et al.: Renin-angiotensin-aldosterone system in patients with sleep apnoea: prevalence of primary aldosteronism. J Renin Angiotensin Aldosterone Syst. 2010, 11:165-72. 10.1177/1470320310366581 